\title{
A NOVEL CFD APPROACH FOR MODELLING THE HIGH-PRESSURE DIRECT INJECTION AND MIXTURE FORMATION IN A SPARK-IGNITED CNG ENGINE
}

\author{
A. TWELLMEYER ${ }^{1}$, F. KÖPPLE ${ }^{1} \&$ B. WEIGAND ${ }^{2}$ \\ ${ }^{1}$ Robert Bosch GmbH, Germany. \\ ${ }^{2}$ Institute of Aerospace Thermodynamics (ITLR), University of Stuttgart, Germany.
}

\begin{abstract}
The use of compressed natural gas $(\mathrm{CNG})$ as a fuel in internal combustion engines brings significant advantages in terms of reduction of $\mathrm{CO}_{2}$-emissions and fuel consumption. Compared to standard gasoline combustion, the $\mathrm{CO}_{2}$-production can be clearly reduced by using a methane-based fuel as it has a beneficial $\mathrm{H} / \mathrm{C}$ ratio. The high knock resistance of methane allows higher compression ratios so that the thermodynamic efficiency is enhanced. Furthermore, the realization of a stratified mixture formation concept shows great potential to further increase fuel savings due to the reduced throttling losses.

In the present work, an URANS-based simulation strategy using the commercial code AVL Fire for the direct injection (DI) of CNG and the mixture formation, including a discretisation of the full nozzle and cylinder geometry is presented. High pressure ratios between the injector and the cylinder lead to a choked flow inside the nozzle. A supersonic region, including shock-occurrence follows as the jet is expanded further downstream the orifice. To successfully resolve the multi-scale flow phenomena the mesh generation process involves the design of a fine hexahedral mesh for the injector, which is merged to the moving cylinder mesh by an arbitrary interface. Turbulence is modelled using the $k-\zeta-f$ model. To estimate the grid-induced error of the simulation, a set of calculations was performed on meshes of decreasing cell dimension. Different nozzle geometries are investigated and evaluated with regard to their mixture formation suitability as well as the effect of increasing rail pressure. Variations include an inward opening multi-hole injector and an outward opening annular ring injector. The results show a strong tendency of the gas jets to interact with each other and with the surrounding walls.

Keywords: compressed natural gas, compressible flow, direct injection, internal combustion engines, stratified mixture formation, supersonic gas jet.
\end{abstract}

\section{INTRODUCTION}

Compressed natural gas $(\mathrm{CNG})$ has become a promising alternative to conventional petroleum-based fuels for internal combustion engines in passenger cars. Due to stricter emission legislations, attention has been drawn to alternative fuels such as methane whose combustion is lean in carbon dioxide emissions. The shorter chain length of the $\mathrm{CH}_{4}$-molecule leads to a higher octane number whereby the compression ratio of the engine can be increased. This results in a higher thermodynamic efficiency, enabling the realization of lower fuel consumption rates. The stratified lean combustion with direct injection (DI) provides further saving potential because a quality-management of the fuel-air mixture allows operation at wideopen throttle with much lower gas exchange losses. In addition to this, excess air results in a higher isentropic exponent of the mixture and has a positive impact on the thermodynamic efficiency of the process [1]. It was shown that a stratified lean DI operation mode of a spark-ignited CNG engine consumes significantly less fuel than a stoichiometric homogeneous port fuel injection (PFI) operation mode [2].

A major challenge of the combustion system development is the investigation of a suitable gas jet guidance inside the cylinder, whereby an ignitable mixture is located near the spark plug at ignition time. The lower volumetric calorific value of gaseous CNG compared to 
liquid fuels requires higher injection rates to ensure sufficient energy intake into the cylinder. Therefore, high rail pressures and large cross-sections of the injector are beneficial parameters to take into account during the development process. As the gas jet behaves differently depending on the operating point and the nozzle geometry, URANS based three-dimensional CFD simulation is an appropriate mean to explore optimal properties of the mixture formation. The numerical simulation of a supersonic gas jet emerging from a high-pressure reservoir through a nozzle is a challenging task. High gradients of the flow values occur as the gas undergoes a sudden expansion and shock waves are formed further downstream the orifice. To properly capture the supersonic flow structures in the vicinity of the injector outlet, a fine grid is required. As the outer dimensions of the cylinder bore are by several orders of magnitudes larger than the nozzle, it is a difficult task to find a suitable compromise for an adequate local grid refinement level. The resulting computational demand is a major restriction for simulating the transient process of the supersonic injection and the mixture formation.

Several simplifying approaches to model the gas injection have been presented to avoid a high grid resolution at the nozzle orifice. Some authors have adopted the approach of a virtual injector model where the injector is not discretized but replaced by a supersonic inlet boundary condition. Additional information about the internal nozzle flow has to be provided by preliminary detailed computations [3] or analytical approaches [4] to determine the inlet flow parameters or the additional source terms. Their aim is to compensate for the non-existent internal injector geometry as well as the small number of grid points across the inlet. With rising available computational resources, the trend is going towards using substitute geometries such as cylindrical shapes for the injector and includes them in the engine mesh $[5,6]$. This is a suitable method that can well reproduce supersonic flow pattern depending on the mesh resolution, but it doesn't provide much flexibility in varying the injector geometry. A different approach to simulate the gas injection was adapted from the well-established liquid fuel spray injection simulation in which droplets are replaced by gaseous spheres and introduced into the computational domain by mass source terms $[7,8]$. Its main advantage is the small modelling effort because no discretisation has to be made for the nozzle. However the turbulent mixing within these jets is underestimated because no shockwaves are present in the result.

It is shown in this work that the flow pattern of gas jets from complex injector geometries is subject to significant variation in behaviour which depends on the local cell size and the operating conditions. A sufficient mesh resolution therefore is necessary to meet demands on accuracy. The objective of the work is to present a novel approach to simulate the direct gas injection. This takes account of local requirements on grid spacing and provides the flexibility to vary injector geometries and injection timing without modifying the moving cylinder mesh.

\section{METHODOLOGY}

In the presented work two different injector types have been examined with regard to their mixture formation ability:

- Injector A being an inward opening multi-hole injector with seven nozzle bores. One of them has a larger diameter of $0.7 \mathrm{~mm}$ and is centrally located on the injector tip. The other six bores are laterally placed around the injector tip and have a diameter of $0.18 \mathrm{~mm}$. Two of the lateral nozzle holes are pointing towards the spark plug and the other four are targeted into the combustion chamber at a steeper angle.

- Injector B is an outward opening annular ring injector whose cross-section has a width of $0.06 \mathrm{~mm}$ and a diameter of $3.8 \mathrm{~mm}$. 
A pre-study on a reduced geometry of the injector A has been performed to determine an adequate grid spacing and compare the numerical results to experimental data obtained from planar laser-induced fluorescence (LIF) inside an optical accessible injection chamber. The simulations were performed using the commercial code AVL Fire on a medium-sized hexahedral static grid, including the discretized injector head inside the flow region. Additionally a highly-resolved calculation was carried out for the near-field of the gas jet using the commercial code Ansys CFX on a dynamic hexahedral mesh, including a part of the internal nozzle geometry and the needle movement.

Thirdly, Fire was used to analyse the mixture formation for injector A and B inside the cylinder utilizing a grid with local refinements and mesh movement considering the variable piston position. In this case, two different meshes, one of mixed type for the cylinder and one hexahedral mesh for the injector-region, were generated and merged by an arbitrary interface. This way a separate block-structured grid, including necessary refinements and boundary layers inside the nozzle can be created solely for the supersonic region. All simulations referenced in this work treat the injection of pure methane into the air, whereas in the LIF experiments methane is injected into pure nitrogen. Deviations of the experimental results due to the missing oxygen content in the surrounding air were found to be small and will be neglected in the comparison of numerical and experimental results.

\section{PRE-STUDY}

\subsection{Internal nozzle flow}

As gas is expanded from a high pressure reservoir into surrounding air, supersonic conditions are reached at the narrowest cross-section of the nozzle as the ratio between the total pressure inside the reservoir and the static pressure at the throttling point becomes critical [9]. The narrowest cross-section of injector A is located at the needle seat. The flow is then bent to either emerge the injector from one of the lateral holes or the central nozzle bore, resulting in a complex and asymmetric flow-structure inside the injector. After the deflection around the sharp edges into the nozzle holes the flow separates and recirculation zones are formed at the inlet of each of the bores. The flow structures outside the nozzle vary according to the counter pressure and the air density inside the cylinder. Referring to the engine cycle where flow conditions vary strongly, the gas jet shape is therefore depending on the operating point and changes over time. For an early start of injection (SOI) where the cylinder pressure is still low, an underexpanded gas jet, including a Mach disk as depicted in Fig. 1 on the left is formed from the central bore. As the piston rises, counter pressure increases and the gas jet becomes slimmer (Fig. 1, right).

To create a high-resolved reference simulation, a hexahedral mesh with a mean cell size of $10 \mu \mathrm{m}$ was generated of a small geometry selection, including the injector head and $3 \mathrm{~mm}$ of the flow field downstream the nozzle outlet. Due to slow convergence of Fire calculations the solution on the large mesh was obtained by using CFX. The shear stress transport (SST) model was used to calculate turbulence effects and real gas behaviour was taken into account by applying the Peng-Robinson equation of state. As a result of the large velocity gradients between the supersonic jets and the surrounding gas, air is entrained within the shear layer and transported with the jet by advection. This leads to the formation of a low pressure area in between the jets, which is illustrated in Fig. 2 (left) on a horizontal cut through the flow field $1 \mathrm{~mm}$ underneath the injector tip. The induced velocity field is shown on the right. 

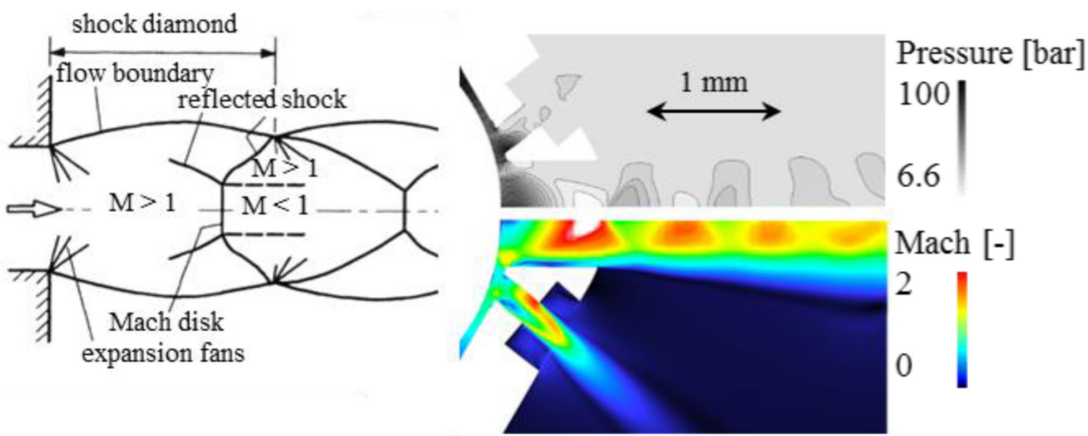

Figure 1: Theory (left): Rotationally symmetric underexpanded high-pressure free gas jet [9]. Simulation (right): Pressure and velocity profile of gas jet emerging from injector $\mathrm{A}$ for top dead centre conditions. $\mathrm{p}_{\text {Rail }}=100 \mathrm{bar}, \mathrm{p}_{\mathrm{Cyl}}=21.26 \mathrm{bar}, \mathrm{T}_{\mathrm{Air}}=750$ $\mathrm{K}, \mathrm{t}=1 \mathrm{~ms}$ after $\mathrm{SOI}(\mathrm{CFX}, 10 \mu \mathrm{m}$ mesh spacing).

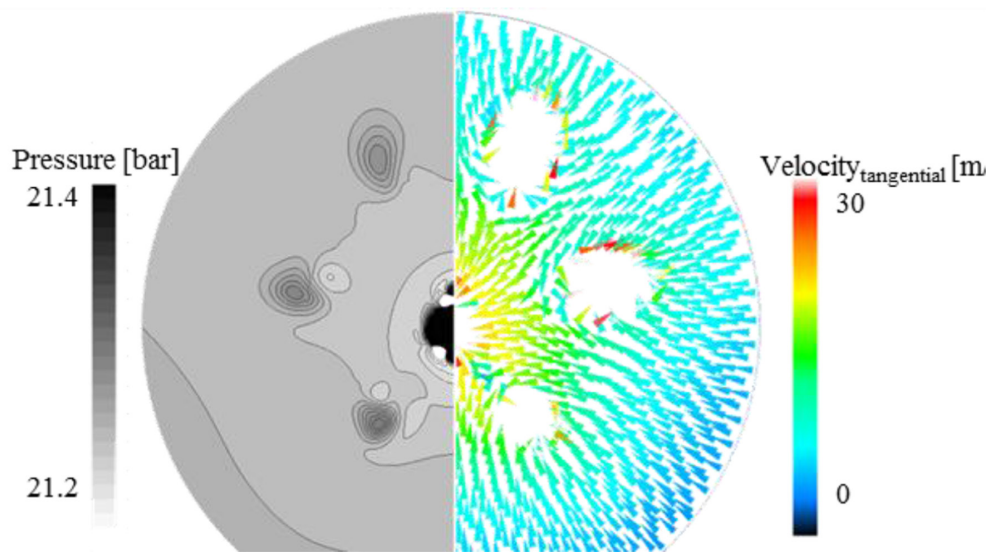

Figure 2: Pressure and tangential velocity on horizontal cut $1 \mathrm{~mm}$ downstream the nozzle orifice (Injector A) for top dead centre conditions. $\mathrm{p}_{\text {Rail }}=100 \mathrm{bar}, \mathrm{p}_{\mathrm{Cyl}}=21.26 \mathrm{bar}$, $\mathrm{T}_{\text {Air }}=750 \mathrm{~K}, \mathrm{t}=1 \mathrm{~ms}$ after SOI (CFX, $10 \mu$ m mesh spacing).

Because of the comparatively large cross-section of the central nozzle bore, its jet has a higher momentum than the surrounding jets. The tangential velocity vectors are pointing towards the centre not only indicating the air entrainment on the one hand but also the attraction of the lateral jets towards the central jet. This effect is increasing as rail pressure and penetration velocity rise. The lateral gas jets will eventually collide into the central jet if the pressure ratio exceeds a critical value.

\subsection{Gas injection into still air}

To investigate the behaviour of the gas jets and their interaction further downstream the injector, a larger hexahedral mesh was created to cover $40 \mathrm{~mm}$ of the flow region. The mesh was coarsened so that the cell size in the nozzle region is about $20 \mu \mathrm{m}$ and increases continuously 
towards the lower edge. The calculations were performed using Fire assuming an ideal gas behaviour in combination with the $k-\zeta-f$ turbulence model. The latter is an eddy-viscosity model, which contains an additional transport equation for the wall-normal velocity scale ratio instead of using empirical damping functions to reproduce the flow in the boundary layer. Therefore, the deficiencies observed with the standard $k-\varepsilon$ model in the near-wall region are avoided [10].

The simulation results show that supersonic structures are still being resolved but less sharp than in Fig. 1 (right). Due to the coarser mesh, the number of reproduced shock waves decreases. Depending on the pressure ratio the jet angle varies and even collides into one single central gas jet. To validate the prediction of the simulation, planar LIF-measurements of the injection were carried out at an optical accessible injection chamber. The chamber was continuously fed by pressurized and heated nitrogen to attain the desired surrounding conditions. Toluene was mixed into methane to visualise the gas jets on the vertical laser light sheet. At fixed surrounding conditions, the lateral jets are colliding into the central jet for increasing pressure ratios as indicated in Fig. 3. These tendencies of the fully developed gas jets compare well between the experiment and the simulation results. For the LIF results the colour bar indicates the fluorescence intensity detected by the camera, which varies with temperature. Thus, the LIF intensity is not proportional to the methane mass fraction put out by the simulation but serves as an adequate indicator to compare the overall jet shape.

\section{IN-CYLINDER SIMULATION}

The major difficulty to set up a CFD simulation for a gaseous injection at real engine dimensions and time scales is the trade-off between the required accuracy and the available computing time. It was predicted in the pre-study that the accurate calculation of the veloc-

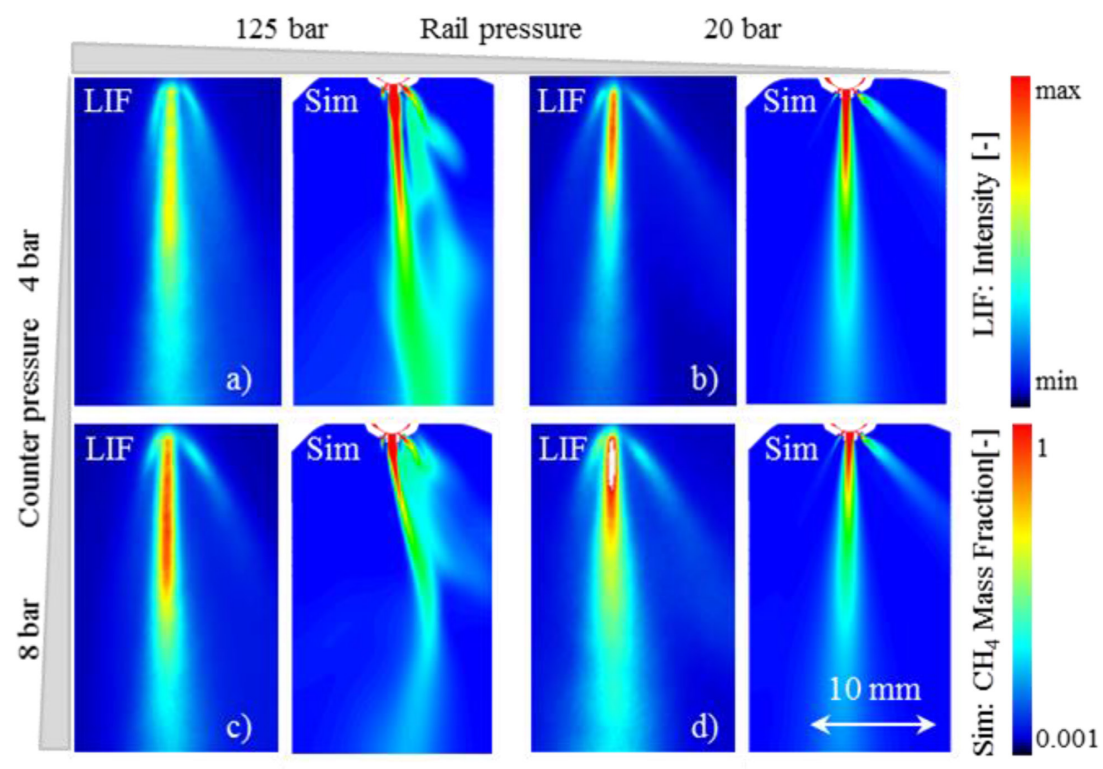

Figure 3: Gas jet from injector A at $\mathrm{t}=2 \mathrm{~ms}$ after SOI (a) + (b) air density $\rho_{\text {Air }}=3.3 \mathrm{~kg} / \mathrm{m}^{3}$ (c) + (d) $\rho_{\text {Air }}=5.3 \mathrm{~kg} / \mathrm{m}^{3}$. Experimental results from LIF (left) and simulation results using Fire (right), mesh: 1.8 million cells. 
ity field has a substantial influence on the general jet behaviour. Figure 4 demonstrates the influence of the spatial resolution on the angle between the lateral jet pointing towards the spark plug and the central jet. The finer mesh (right) results in a significantly wider opening angle so that the lateral gas jet is detached from the central one and approaching closer to the spark plug where it results in a locally richer mixture. To evaluate variations in mixture quality, the local equivalence ratio, which is the inverse of the air-fuel ratio, is depicted in Fig. 4.

A suitable meshing procedure for the whole engine simulation was found in splitting the geometry into two segments to meet different requirements for each part. A static blockstructured hexahedral mesh was generated for a small cut-out of the geometry, including the injector as indicated in Fig. 5. Thus, a cell size of $30 \mu \mathrm{m}$ can be provided near the outlet and boundary layers are included in each of the nozzle bores. Flow-oriented hexahedral meshes have proven to be the appropriate choice to simulate supersonic nozzle flows due to stability reasons. Experience has also shown that a slight coarsening of the mesh in the near-nozzle region is an effective measure to prevent simulation start-up problems of Fire runs. In the second step the surrounding engine geometry is discretised by a set of mov-

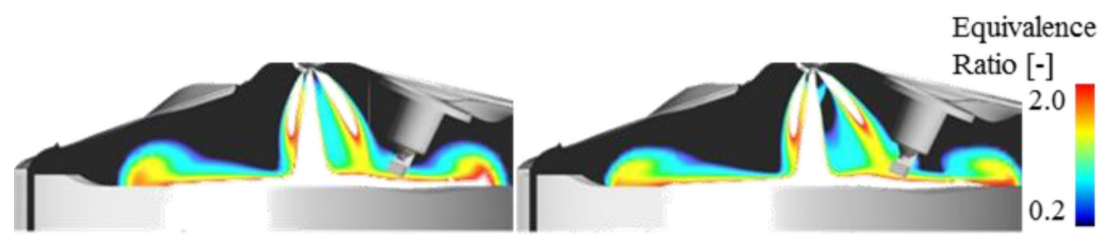

Figure 4: Jet behaviour emerging from injector A for increasing mesh resolution: 4.5 million cells (left) and 23.6 million cells (right). Conditions at top dead centre $\mathrm{p}_{\text {Rail }}=100$ bar, $\mathrm{p}_{\mathrm{Cyl}}=21.26$ bar, $\mathrm{T}_{\text {Air }}=750 \mathrm{~K}, \mathrm{t}=1.8 \mathrm{~ms}$ after $\mathrm{SOI}(\mathrm{CFX})$.

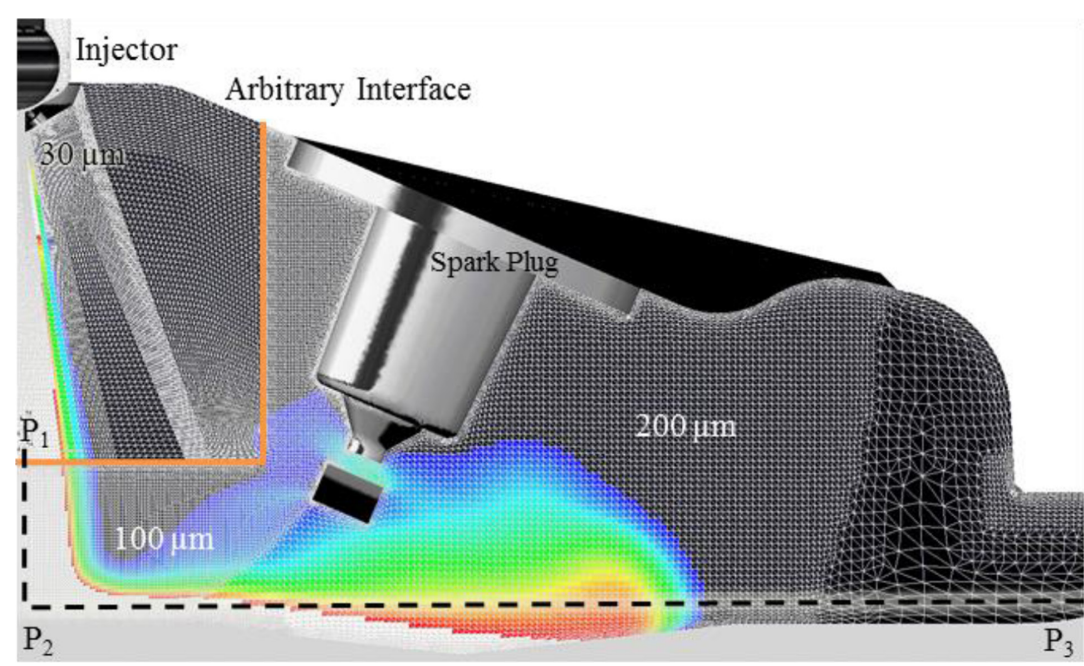

Figure 5: Refinement levels of the mesh (13.5 million cells) used for the in-cylinder calculations. 
ing meshes of mixed type. Two spherical refinements of $100 \mu \mathrm{m}$ and $200 \mu \mathrm{m}$ in cell size are placed around the injector position. Both meshes are merged by creating an arbitrary interface, which establishes the mathematical connection between the two non-conformal domains. The joined mesh adds up to a total number of 13.5 million cells. An operating point at part load with 2,000 rpm and 4 bar mean indicated pressure was referenced to perform the engineering calculations. The injection starts at $27.5^{\circ}$ crank angle (CA) before top dead centre (BTDC).

A pressure inlet boundary was chosen for the injector inflow condition. The injection was calculated with injector A and B for two different rail pressures each. Fire was used to perform the engineering calculations applying an ideal gas equation of state and the $\mathrm{k}-\zeta$-f model for turbulence.

\subsection{Multi-hole injector}

Figure 6 shows the mixture formation in the vertical symmetry plane and on a horizontal cut through the electrodes of the spark plug for two different points in time. The doubling of rail pressure results in a richer core and higher air entrainment. Even though the total amount of ignitable mixture increases with rail pressure, the relative ignitable mixture fraction per unit

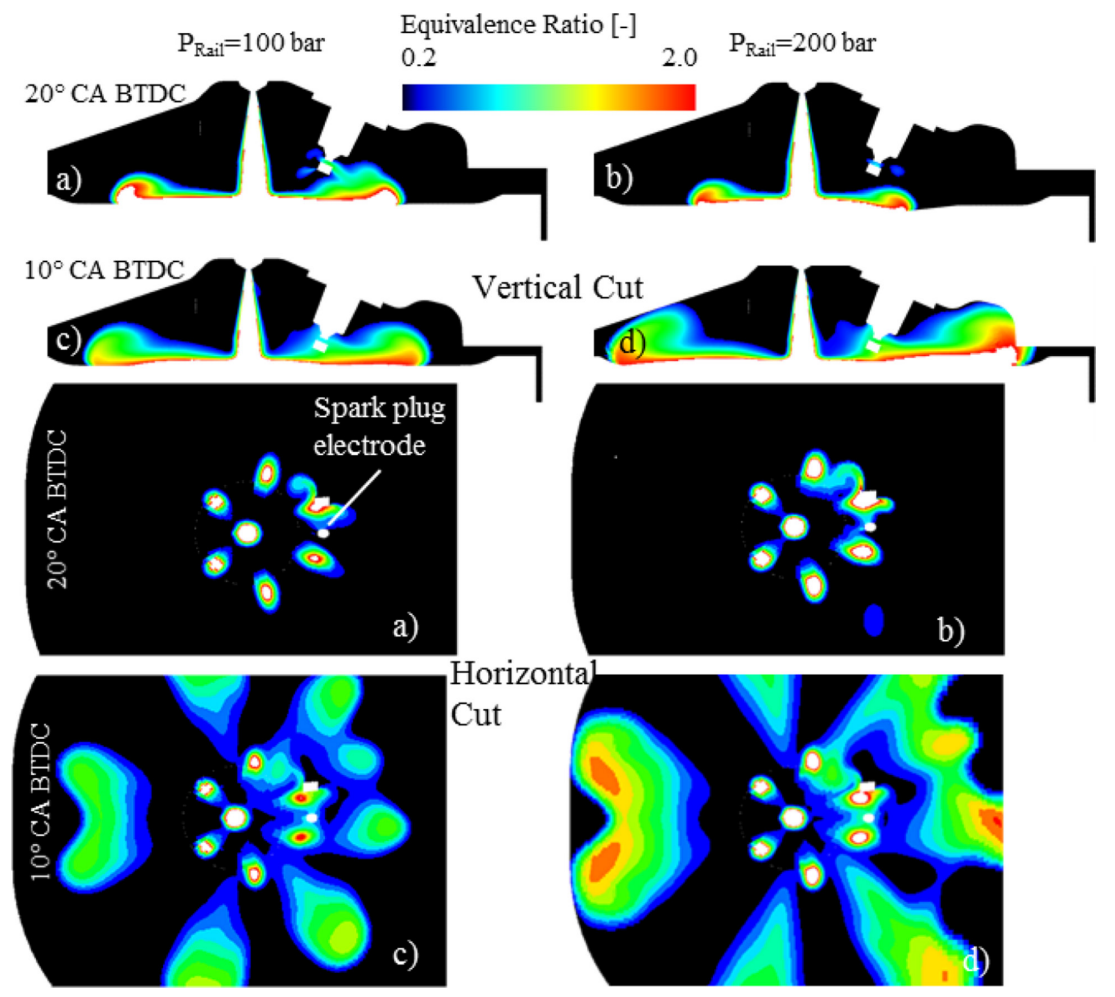

Figure 6: Mixture formation with Injector A for two different rail pressures and times: $\mathrm{p}_{\text {Rail }}=$ 100 bar (a) \& (c) and $\mathrm{p}_{\text {Rail }}=200$ bar (b) \& (d); t $=20^{\circ}$ CA BTDC (a) \& (b) and $\mathrm{t}=$ $10^{\circ}$ CA BTDC (c) \& (d). Performed on mesh with 13.5 million cells. 
mass injected drops. The higher rail pressure, therefore, leads to a wider distribution of lean mixture throughout the cylinder and the formation of rich zones inside the jet cores. Due to the reflection of the gas jet on the piston and the approaching of two lateral gas jets at the spark plug a suitable mixture quality is ensured over the whole range of possible ignition times (cf. Fig. 7).

\subsection{Annular ring injector}

Annular ring injectors are attractive to use for the gaseous DI because they have larger cross-sections and can ensure higher mass flows than common multi-hole injectors. This is of special interest since CNG has a lower volumetric energy content than liquid fuels. The narrowest cross-section of injector B is located at the nozzle opening where the speed of sound is reached. The gas jet from the annular ring injector is characterised by strong differences in shape depending on the operating point as indicated in Fig. 8. With an increasing pressure ratio, the jet will either form a hollow cone as in (a) + (c) or follow the contour of the surrounding walls of the cylinder head as in (b) + (d). In line with the findings from the multi-hole jets, the injection from an annular ring injector induces a low pressure area around the jet itself causing it to be attracted by the walls. This leads to strong local differences in mixture quality. In the case where the gas jet forms a hollow cone, it is pointing directly at the spark plug so that the mixture is too rich to be ignited.

In the second case where the jet sticks to the wall, almost no methane reaches the spark plug resulting in a locally very lean mixture. Figure 7 summarizes the results from the in-cylinder simulation. The local equivalence ratio at the spark plug is indicated over time. The multi-hole injector achieves an appropriate mixture formation over a long period of time irrespective of the injection pressure. It shows great potential for the application in CNG engines with stratified mixture formation. The annular ring injector on the other hand has to undergo further modifications to ensure a suitable mixture formation. Its jet shows a strong tendency to stick to the surrounding walls and cannot ensure an ignitable mixture composition around the spark plug.

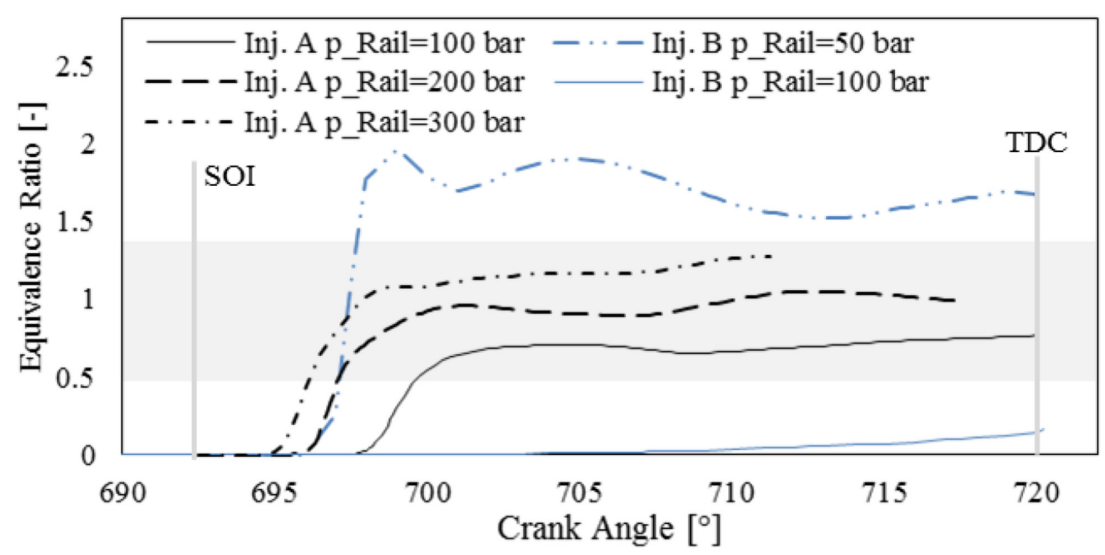

Figure 7: Equivalence ratio at the spark plug for Injectors A and B for different rail pressures. 


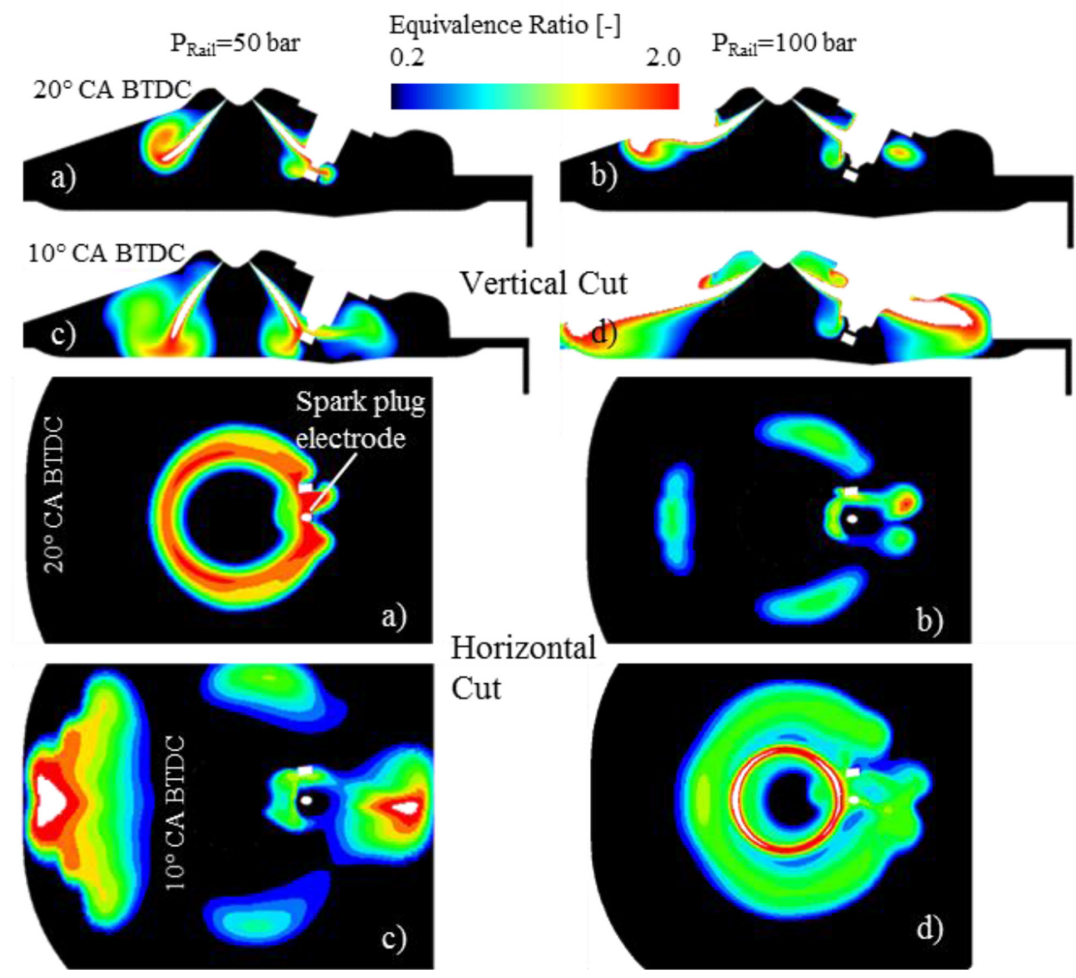

Figure 8: Mixture formation with Injector B for two different rail pressures and times: $\mathrm{p}_{\text {Rail }}=$ 50 bar (a) \& (c) and $\mathrm{p}_{\text {Rail }}=100$ bar (b) \& (d); $\mathrm{t}=20^{\circ} \mathrm{CA}$ BTDC (a) \& (b) and $\mathrm{t}=$ $10^{\circ}$ CA BTDC (c) \& (d). Performed on a mesh with 13.1 million cells.

\section{CONCLUSION}

In this work a novel simulation approach was presented to calculate high pressure gaseous injections under real engine conditions and geometric dimensions. It includes the detailed discretization of the internal injector geometry because the asymmetric flow inside the nozzle influences the jet formation after emerging from the injector. The meshing strategy takes account of the varying requirements of the local resolution and provides high flexibility in exchanging or adapting the injector as well as varying injection timing. It was shown that the results are highly dependent on the degree of spatial resolution to an extent where mesh coarsening may change the overall jet shape. However, using the proposed simulation approach, a good correspondence between the calculated and the measured mixture formation has been shown. Furthermore, the results demonstrate that the gas jets from different injector types are likely to interact with each other or the surrounding walls depending on the pressure ratio between rail and cylinder, resulting in completely different mixture composition around the spark plug. Thus, by using the novel simulation approach shown here, the combustion system development of CNG-DI engines with stratified-charge operation can be supported substantially. 


\section{REFERENCES}

[1] Merker, G.P. \& Schwarz, C., Grundlagen Verbrennungsmotoren, Vieweg + Teubner: Wiesbaden, 2009.

[2] Friedrich, W., Grzeszik, R. \& Wensing, M., Mixture formation in a CNG-DI engine in stratified operation. In SAE Technical Paper, 2015.

[3] Johnson, N.L. \& Amsden, A.A., Three-dimensional computer modeling of hydrogen injection and combustion. In '95 SMC Simulation Multiconference, Phoenix, Arizona, USA, 1995.

[4] Ouellette, P. \& Hill, P.G., Turbulent transient gas injections. Journal of Fluids Engineering, 122, pp. 743-753, 2000. http://dx.doi.org/10.1115/1.1319845

[5] Lucchini, T., D’Errico, G. \& Fiocco, M., Multi-dimensional modeling of gas exchange and fuel-air mixing processes in a direct-injection, gas fueled engine. In SAE Technical Paper, 2011. http://dx.doi.org/10.4271/2011-24-0036

[6] Yadollahi, B. \& Boroomand, M., The effect of combustion chamber geometry on injection and mixture preparation in a CNG direct injection SI engine. Fuel, 107, pp. 52-62, 2013. http://dx.doi.org/10.1016/j.fuel.2013.01.004

[7] Hessel, R.P. and Abani, N., Gaseous fuel injection modeling using a gaseous sphere injection methodology. In SAE Technical Paper, 2006. http://dx.doi.org/10.4271/2006-01-3265

[8] Chiodi, M., Berner, H.J. \& Bargende, M., Investigation on different injection strategies in a direct-injected turbocharged CNG-engine. In SAE Technical Paper, 2006.

[9] Rist, D., Dynamik Realer Gase, Berlin Heidelberg: Springer, 1996. http://dx.doi.org/10.1007/978-3-642-61402-6

[10] Hanjalic, K., Popovac, M. \& Hadziabdic, M., A robust near-wall elliptic-relaxation eddy-viscosity turbulence model for CFD. International Journal of Heat and Fluid Flow, 25, pp. 1047-1051, 2004.

http://dx.doi.org/10.1016/j.ijheatfluidflow.2004.07.005 